\section{Risco de adoecimento por exposição às águas do Rio Doce: um estudo sobre a percepção da população de Tumiritinga, Minas Gerais, Brasil}

\author{
Risk of contamination from exposure to Rio \\ Doce water: a case study on the population's \\ perceptions in Tumiritinga, Minas Gerais State, \\ Brazil
}

\author{
Riesgo de enfermedades por la exposición a \\ las aguas del Río Doce: un estudio sobre la \\ percepción de la población de Tumiritinga, \\ Minas Gerais, Brasil
}

Gilvan Ramalho Guedes 1

Andréa Branco Simão 2

Carlos Alberto Dias 3

Eliza de Oliveira Braga 4

\author{
${ }_{1}^{1}$ Faculdade de Ciências \\ Econômicas, Universidade \\ Federal de Minas Gerais, Belo \\ Horizonte, Brasil. \\ 2 Escola de Serviço Social, \\ Pontifícia Universidade \\ Católica de Minas Gerais, \\ Belo Horizonte, Brasil. \\ 3 Faculdade Interdisciplinar \\ em Humanidades, \\ Universidade Federal dos \\ Vales do Jequitinhonha e \\ Mucuri, Diamantina, Brasil. \\ 4 Universidade Federal de \\ São João del-Rei, São João del \\ Rei, Brasil. \\ Correspondência \\ G. R. Guedes \\ Departamento de \\ Demografia, Faculdade \\ de Ciências Econômicas, \\ Universidade Federal de \\ Minas Gerais. \\ Av. Antônio Carlos 6627, \\ Belo Horizonte, $M G$ \\ 31270-901, Brasil. \\ grguedes@cedeplar.ufmg.br
}

\begin{abstract}
The close relationship between local residents and the Rio Doce and the river's recurrent flooding lead to continuous exposure of the popula tion to waterborne diseases. Given the epidemiological importance of such diseases in the region, this study analyzes the association between risk perception of contamination and river water use, as well as the heuristic mechanisms used by individuals to shape their personal perception of risk. Regression models coupled with thematic network analysis were applied to primary data from 352 households in 2012. The data are representative of urban residents of Tumiritinga, Minas Gerais State, Brazil. The results show that while $92.6 \%$ of respondents perceived high risk of waterborne diseases, only $11.4 \%$ reported not making direct use of the river. This apparent paradox is explained by the lack of information on transmission mechanisms, underestimating the perception of contamination. Public campaigns to promote preventive behavior should stress how waterborne diseases are transmitted, using simple examples to reach a wider local audience.
\end{abstract}

Environmental Risks; Water Pollution; Waterborne Diseases

\section{Resumo}

A forte relação das pessoas com o Rio Doce, bem como suas enchentes regulares, representam fator contínuo de exposição ao risco de doenças hídricas. Dada sua relevância epidemiológica, este estudo analisa a associação entre percepção de contaminação e uso do rio, bem como os mecanismos heurísticos empregados na formação da percepção de risco. Utilizou-se um modelo probit ordenado com variável instrumental e análise de redes temáticas aplicados a uma base de dados primária de 352 domicílios, representativa dos moradores de Tumiritinga, Minas Gerais, Brasil, para o ano de 2012. Os resultados indicam que embora a maioria (92,6\%) dos moradores de Tumiritinga perceba o risco de contrair algum tipo de doença quando nada no Rio Doce, somente 11,4\% informam não entrar na água. A análise de conteúdo sugere que esse paradoxo advém da falta de compreensão populacional sobre os mecanismos de transmissão de doenças hídricas, criando viés otimista sobre as chances de contaminação. Campanhas para promoção de comportamento preventivo devem, portanto, enfatizar as formas de contrair doenças hídricas na região.

Riscos Ambientais; Poluição da Água; Doenças Transmitidas pela Água 


\section{Introdução}

A qualidade dos recursos hídricos tem um impacto direto sobre a saúde dos indivíduos 1 . Esse impacto está associado ao padrão de uso das águas, apresentando efeitos deletérios sobre a saúde do usuário e de seus familiares, particularmente quando indicadores socioeconômicos não são favoráveis 2 .

A teoria de risco sugere que a existência de um perigo só se configura como risco se efetivamente conectada à presença humana 3,4 . As propostas recentes de gestão pública da qualidade dos recursos hídricos 5 reconhecem as evidências científicas de que o comportamento de risco individual em relação ao uso de águas contaminadas é influenciado por restrições socioeconômicas, viés otimista na percepção sobre risco de contaminação 6,7 e baixa efetividade da divulgação puramente quantitativa de estatísticas epidemiológicas na promoção de comportamento preventivo 8,9.

Quando iniciativas públicas de eliminação das fontes geradoras de contaminação não existem ou são incompletas, entender os mecanismos que influenciam a percepção de risco é um pré-requisito básico para indução de comportamento preventivo, como sugerido por teorias psicológicas e de saúde sobre comportamento de risco 10,11. Teorias psicológicas sobre susceptibilidade a perigos apontam para o papel mediador que a qualidade da percepção, o envolvimento emocional e a seletividade dos processos cognitivos relativos aos fatores de risco exercem sobre a existência real de um perigo 10,12. Segundo essas teorias, a existência do perigo em si não é suficiente para afetar a percepção sobre a susceptibilidade a ele. Para que haja susceptibilidade, é necessário haver exposição direta, baixa capacidade de resposta e outros mecanismos comportamentais e cognitivos que funcionem como fatores predisponentes.

Estudos laboratoriais e amostrais sugerem que a exposição a comportamentos arriscados é maior em indivíduos que possuem falhas cognitivas na interpretação real do risco, na extensão dos danos potenciais e na compreensão dos mecanismos de geração dos danos 8,13. Weinstein 6 , por exemplo, verificou que indivíduos apresentam viés otimista em relação à susceptibilidade a vários perigos, como doenças sexualmente transmissíveis e violência. Esse viés seria explicado pelo maior peso dado aos fatores de risco de elevada controlabilidade, como ações pessoais e traços psicológicos. Assim, em situações que as pessoas acreditam ter maior controle sobre suas ações, a percepção de risco de contaminação é sistematicamente subestimada, como no caso de alcoolismo, vício em drogas, acidentes de carro e contaminação por doenças venéreas 6,7,10. Embora robustos, os resultados ignoram situações em que há falhas heurísticas sobre o mecanismo de conversão de riscos em danos efetivos. Parte do efeito de controlabilidade, portanto, pode advir da incapacidade dos indivíduos de formular a correta relação entre exposição, mecanismos de causação e consequências 14 .

Outros estudos experimentais sugerem que há um forte componente emocional e relacional na percepção sobre risco e susceptibilidade 8,13 . Por exemplo, indivíduos têm maior chance de classificarem-se como menos expostos a risco que seus pares 6 , embora casos narrativos de contaminação ou dano sofrido por pessoas próximas reduzam o viés otimista sobre a susceptibilidade, induzindo comportamentos preventivos 8 .

O componente emocional da percepção de risco (risk as feeling) parece ser robusto a influências culturais 9 . Estudos realizados em diferentes contextos e sob diferentes condições experimentais sugerem que as experiências diretas e indiretas com o risco influenciam tanto a percepção de risco da ocorrência de um evento quanto o comportamento de exposição a ele. Por exemplo, estudos mostram que pessoas que tiveram conhecidos afetados por alguma doença em sua localidade têm maior probabilidade de adotar medidas preventivas, assim como de dar suporte a políticas governamentais desenhadas para minimização de risco, mesmo que estas ações públicas requeiram algum sacrifício econômico 15,16.

Apesar da ampla literatura sobre percepção de risco, afeição, cognição e comportamento de risco ambiental e de saúde em países desenvolvidos, pouco se conhece sobre estas associações em países em desenvolvimento. Uma importante exceção são os estudos comportamentais sobre risco de contaminação, comportamento sexual e aderência a tratamentos relativos a doenças sexualmente transmissíveis na África 17 e Ásia 18. Para a área de ambiente e saúde, dois estudos para o México também devem ser destacados como raros exemplos dessa literatura na América Latina: Corral-Verdugo et al. 19 e Morua et al. 16.

Estudos sobre risco de contaminação por doenças hídricas no Brasil têm enfatizado o componente epidemiológico relacionado a riscos objetivos 20 , assim como o aspecto de gestão pública dos recursos hídricos 21,22. Pouco se conhece sobre a heurística populacional relacionada aos mecanismos de exposição e percepção de contaminação por doenças hídricas no país. Os estudos epidemiológicos e de saúde sobre doenças hídricas em geral assumem que o processo de decisão individual ocorre com informação com- 
pleta, ignorando o potencial viés heurístico na associação percebida entre chance de contaminação e mecanismos científicos de transmissão de doenças 23 .

Este trabalho tem como objetivo central analisar a influência do uso para consumo e da contaminação de familiares ocasionada pelo uso do Rio Doce sobre o risco percebido de contaminação individual. Adicionalmente, procurou-se analisar as explicações dadas pelas pessoas sobre por que acreditam estar expostas ou protegidas do risco de contaminação por doenças de veiculação hídrica ao entrar no Rio Doce. Para tanto, utilizou-se uma combinação de metodologias quantitativas e qualitativas com vistas a dar resposta a um aparente paradoxo: por que os indivíduos continuam fazendo uso de águas poluídas, mesmo reconhecendo uma alta chance de contaminação? A variável "uso do rio", portanto, é utilizada como uma proxy para comportamento preventivo, dada a alta prevalência de reconhecimento sobre o nível de poluição do Rio Doce entre os entrevistados.

\section{Qualidade da água na bacia do Rio Doce}

A bacia hidrográfica do Rio Doce está situada na região sudeste do país e abriga o maior complexo siderúrgico da América Latina 24. Apesar da concentração de importantes complexos industriais como a Usiminas, Vale do Rio Doce, Belgo Mineira, Acesita e Cenibra, grande parte dos empregos locais nos municípios de menor porte advém das atividades agropecuárias, como no caso de Tumiritinga, Minas Gerais, Brasil. A intensa atividade industrial, associada a uma agropecuária extensiva, gerou um quadro preocupante de erosão, assoreamento dos leitos dos rios, desmatamento e poluição dos recursos hídricos 25 . Por essa razão, o Instituto Mineiro de Gestão das Águas (IGAM) identificou a erosão e o lançamento de esgotos sanitários como os dois principais fatores de pressão associados aos indicadores de degradação da qualidade da água da bacia 22,24,26.

A qualidade da água na bacia hidrográfica do Rio Doce varia ao longo de sua extensão, embora os níveis médios de salubridade sejam bastante precários em toda a sua cobertura geográfica. $\mathrm{O}$ relatório do Ministério da Saúde sobre vigilância da água para o consumo sugere que a presença de alumínio na porção mineira do Rio Doce excede em $97 \%$ o nível máximo preconizado (20\%), seguido pela presença de fosfato total (73\%), coliformes fecais $(64,9 \%)$ e totais $(55,1 \%)$ e índice de fenóis (33\%). A forte concentração de alumínio não está diretamente associada aos dejetos industriais, embora contribua para intensificar o processo de erosão e enchentes ${ }^{22}$. Os demais parâmetros de contaminação (coliformes e fenóis) indicam claramente o papel dos dejetos sanitários, contribuindo para a piora do Índice de Qualidade da Água (IQA) nos últimos anos. O Município de Tumiritinga, por exemplo, tem somente $10,86 \%$ de seu esgoto tratado, gerando um nível de poluição remanescente na água muito acima do ideal.

Aliado ao quase inexistente sistema de tratamento de esgotos, o sistema de uso do solo adotado direta ou indiretamente pelas mineradoras, siderúrgicas e produtoras de celulose contribuiu para intensificar o assoreamento dos leitos do rio. O índice alto de concentração de fosfato nas águas do Rio Doce, por exemplo, necessário para corrigir o solo empobrecido na plantação de eucalipto da região, é um indicador dos efeitos indiretos da demanda industrial por celulose e combustível vegetal. Associado a isso, verifica-se o uso de agrotóxicos de maneira indiscriminada nas lavouras, com aumento significativo da contaminação da água 27.

As mudanças ambientais observadas na região, e em particular o assoreamento intenso do Rio Doce, contribuíram para a formação de uma praia de rio em Tumiritinga. A Prainha do Jaó, como é conhecida, tornou-se um ponto central do município, fornecendo atividades de lazer e turismo, abrigando festividades locais e atraindo recursos durante o período de Carnaval 26. Devido à má qualidade das águas do Rio Doce, o uso intenso da Prainha, incluindo uso para consumo, pesca e banho, gera um fator de exposição epidemiológica para os residentes e turistas 26. Essa exposição voluntária, como será visto adiante, advém de crenças populares sobre a separação entre o rio, a fragilidade do corpo e os mecanismos de transmissão de doenças hídricas. Nesse sentido, o problema é mais de ordem heurística do que de credibilidade da informação oficial sobre riscos 26,27. Entender, portanto, a formação dos mecanismos de percepção de risco e os fatores associados ao comportamento preventivo em relação ao padrão de uso de águas contaminadas é uma questão epidemiológica fundamental para o contexto da região.

\section{Metodologia}

Este artigo utiliza os dados primários oriundos do projeto Representações Sociais das Comunidades de Galiléia e Tumiritinga com Relação ao Uso e Conservação do Rio Doce, financiado pela Fundação de Amparo à Pesquisa do Estado de Minas Gerais (FAPEMIG; Processo no SHAAPQ-01807-11), que teve como principal objetivo entender as representações sociais e as 
formas de uso e conservação do Rio Doce entre os residentes de Tumiritinga e Galiléia, em Minas Gerais. A pesquisa foi feita por integrantes do Grupo de Pesquisa Saúde, Indivíduo e Sociedade (SAIS), da Universidade Vale do Rio Doce. O uso de questionários semiestruturados permitiu a coleta de dados quantitativos e qualitativos padronizados para as duas comunidades, com questões fechadas e abertas aplicadas a todos os indivíduos selecionados na amostra. Os questionários contêm questões relativas a características sociodemográficas dos residentes de Tumiritinga e Galiléia, de uso e conservação do Rio Doce e itens sobre conhecimento, grau de consciência, atitude e comportamento ambiental.

Neste trabalho serão utilizados os dados de Tumiritinga, coletados entre os meses de dezembro de 2011 a janeiro de 2012. A amostra é representativa dos domicílios urbanos do município. O tamanho mínimo amostral foi baseado num erro máximo de 3\%, com nível de 95\% de confiança e proporção de 0,50 (mais conservadora), com correção para população finita. A amostra total corresponde a 352 domicílios urbanos. Para garantir a correta representatividade populacional, o levantamento dos domicílios foi realizado com base na técnica de amostragem probabilística por conglomerados em três estágios. No primeiro, foram definidos grupos homogêneos de bairros, segundo sua classificação socioeconômica, com base no valor predial médio declarado. No segundo estágio, a distribuição proporcional dos grupos foi utilizada para alocar, em cada grupo, o total de domicílios a serem entrevistados. No terceiro, a quantidade de domicílios por grupo homogêneo foi distribuída de forma aleatória. Em cada domicílio amostrado, selecionou-se a pessoa de referência para responder ao questionário. Caso essa não quisesse ou pudesse dar a entrevista, outra pessoa indicada responderia ao questionário, desde que maior de 18 anos. O peso amostral foi calculado com base no inverso da probabilidade de seleção de cada unidade amostral 28. O estudo foi submetido e aprovado pelo Comitê de Ética em Pesquisa da Universidade Vale do Rio Doce (CEP/UNIVALE 072/10-12).

\section{Análise quantitativa}

Para testar a hipótese de associação entre o uso do rio e a presença de algum parente contaminado sobre a percepção de riscos de contaminação por doenças hídricas, foi utilizado um modelo probit ordenado, com teste de Brant para validação do pressuposto das retas de regressões paralelas. O modelo foi escolhido, pois a variável dependente é representada pela classificação dos entrevistados da probabilidade percebida de contaminação ao utilizar o Rio Doce. Foi feita a seguinte pergunta no questionário: "em sua opinião, quando alguém entra no Rio, qual o risco de que seja contaminado por alguma doença que possa estar na água?". As categorias de respostas dadas foram: 0 - ausência de risco; 1 - pequeno; 2 - médio; 3 - alto. A variável, portanto, implica um ordenamento do risco percebido, exigindo um modelo não linear do tipo ordinal. O teste de Brant não rejeitou a hipótese de que um modelo que assume proporcionalidade de medidas de associação é adequado aos dados do estudo (valor de $\mathrm{p}=0,39$ ). Assim, assume-se que o modelo ordinal é apropriado aos objetivos apresentados. As análises foram realizadas utilizando-se o software Stata MP 13.0 (Stata Corp., College Station, Estados Unidos).

Apesar de tradicionalmente estarmos acostumados a pensar que a percepção de risco induz o comportamento preventivo, pessoas que fazem uso de um rio com águas poluídas podem subestimar a percepção sobre as chances de contaminação 2,12,16. Portanto, "o uso do rio" está sendo usado nesta análise como proxy para comportamento preventivo, uma vez que em estudo anterior 26 , análises descritivas e evidências qualitativas mostraram que há uma alta prevalência de uso, embora seja reconhecido o elevado nível de poluição das águas do Rio Doce.

Esse aparente paradoxo coloca um desafio metodológico, que é a simultaneidade de efeito entre uso e percepção de risco de contaminação. Para minimizar a endogeneidade por simultaneidade, foi utilizada uma estratégia de estimação em dois estágios com variáveis instrumentais 29 . Por variável instrumental entende-se aquela que está relacionada com a variável explicativa endógena (uso do rio), porém não está associada com a variável dependente (percepção sobre contaminação). O instrumento empregado foi a distância de cada domicílio à Prainha do Jaó Essa praia de rio é resultado do processo de assoreamento relativo à eliminação das matas ciliares e do lançamento de resíduos sólidos ao longo do Rio Doce. Ao estudar as representações sociais dos moradores de Tumiritinga quanto à Prainha do Jaó e o Rio Doce, Hora 26 encontrou representações completamente distintas. Enquanto a Prainha era vista como uma imagem positiva, associada ao lazer e turismo, o Rio Doce foi visto como um recurso degradado pelo desmatamento e contaminado por poluição vinda de resíduos industriais e esgotamento sanitário não tratado.

Essa diferença na representação social sugere que a distância até a Prainha do Jaó é um bom candidato a instrumento para corrigir o viés causado pela endogeneidade entre uso e per- 
cepção de risco de contaminação. Foram utilizados dois instrumentos diferentes: a distância reportada no questionário e a distância em linha reta, calculada pela grade georreferenciada dos domicílios entrevistados. A distância reportada em questionário comportou-se melhor do que a distância em linha reta, exatamente por considerar diferenças na dificuldade de acesso. O teste de associação bivariada entre as medidas de distância, uso do rio e chance de contaminação mostrou uma relação estatisticamente significativa (valor de $\mathrm{p}<0,01$ ) entre o instrumento e o uso do rio, mas não apresentou associação significativa (valor de $\mathrm{p}=0,55$ ) entre o mesmo instrumento e a medida de percepção de risco. $\mathrm{O}$ teste de Hausman para endogeneidade na equação do segundo estágio identifica a forte presença de endogeneidade (valor de $\mathrm{p}<0,01$ ), influenciando de maneira significativa o coeficiente de uso na regressão de percepção de contaminação. A qualidade do instrumento do ponto de vista estatístico foi validada pela significância dos coeficientes do instrumento (resultados sob requisição aos autores) na equação do segundo estágio (a equação de uso do rio, utilizando o instrumento como preditor e todas as demais variáveis exógenas do modelo de risco de contaminação).

\section{Análise qualitativa}

Foram analisadas as respostas dadas pelos entrevistados à questão aberta na qual explicam os motivos pelos quais acreditam correr risco de contaminação por alguma doença ao entrarem no rio. A análise foi desenvolvida baseando-se na proposta de Attride-Stirling 30, denominada Análise de Redes Temáticas. De acordo com essa proposta, a análise de redes temáticas inclui seis passos: (i) codificação do material; (ii) identificação dos temas; (iii) construção das redes temáticas; (iv) descrição e exploração das redes temáticas; (v) sumarização das redes temáticas e; (vi) interpretação.

A curiosa relação entre elevada classificação de chance de contaminação e uso intenso do rio verificada nos dados foi escrutinada com base em categorias temáticas que pudessem sinalizar a necessidade do uso, a percepção sobre susceptibilidade e as crenças sobre os mecanismos de transmissão de doenças. Assim, a análise temática permitiu a criação de três grandes grupos: (1) a poluição do Rio Doce; (2) as doenças advindas do Rio Doce; e (3) entrar ou não entrar no rio. Essas categorizações temáticas permitiram qualificar o resultado encontrado nos modelos de regressão de que o uso do rio para consumo induz uma percepção reduzida de classificação de risco (viés otimista).

\section{Resultados}

A amostra original, de 352 domicílios, foi reduzida a um total de 278 observações em razão de informações faltantes para algumas das variáveis utilizadas. De acordo a Tabela 1, as pessoas apresentam um ligeiro viés pessimista sobre a possibilidade de contaminação. Entre os que acreditam no risco de contaminação pelo uso do rio, $53,7 \%$ percebem o risco como alto. Somente $29 \%$ identificam a poluição advinda da água e esgotamento sanitário como principal problema do rio, contrastando com os dados oficiais sobre fontes de poluição 24 . Em média, $81 \%$ fazem uso do rio, a despeito do reconhecimento do risco de contaminação. Metade da amostra já teve algum familiar contaminado por doenças hídricas pelo uso do Rio Doce. A idade dos respondentes variou de 18 a 98 anos, com uma média de 45 anos, sendo $70 \%$ mulheres, com renda domiciliar per capita média inferior a 1 salário mínimo (R\$ 622,00 em 2012). Cerca de $72 \%$ dos entrevistados são residentes no município há mais de 10 anos ininterruptos (destes, 33\% são nativos). O tempo de deslocamento médio até a Prainha do Jaó é de cerca de 16 minutos. Das variáveis analisadas, apenas o uso do rio e a presença de familiar infectado apresentaram associação bivariada estatisticamente significativa com a chance de contaminação.

A Tabela 2 apresenta os resultados dos modelos de regressão. Foram feitos modelos sequenciais, em bloco. O modelo I contém apenas as variáveis estado (uso do rio e familiar infectado); o modelo II introduz variáveis sociodemográficas de controle, e o modelo III adiciona uma variável indicadora (dummy) para a percepção de que esgoto e lixo são os principais problemas ambientais do rio. Para cada bloco são apresentados os resultados do modelo probit ordenado, seguido do modelo em dois estágios com variável instrumental para atenuar o viés de endogeneidade no coeficiente do uso do rio. A presença de um familiar contaminado por doenças hídricas tem um efeito estatístico inconteste sobre a chance de perceber riscos mais elevados de contaminação ao se usar o rio. Esse resultado é coerente com as teorias psicológicas de risco como afeto (risk as feeling). O efeito do uso do rio sobre a classificação percebida de risco é coerente com a teoria de viés heurístico, sugerindo que pessoas que utilizam as águas do rio para consumo tendem a apresentar viés otimista na classificação do risco. A significância do uso, apenas nos modelos de variáveis instrumentais, sinaliza o efeito da endogeneidade entre uso e percepção de risco (Hausman - valor de $\mathrm{p}<0,01$ ). Assim, quem usa o rio para consumo tem chance quase 
6 vezes menor de classificar o risco como alto se comparado a um não usuário (Tabela 2).

$\mathrm{O}$ viés otimista, como indicam os resultados qualitativos, advém não da percepção sobre controlabilidade, como advogam alguns autores, mas pelo viés heurístico sobre os mecanismos de contaminação. Os dados mostram que, entre aqueles entrevistados que entram no rio, 92,6\% acreditam existir algum risco de contaminação e somente $4,8 \%$ alegam acreditar não estarem expostos ao risco de serem contaminados ao entrar na água. Com base nesse resultado é possível inferir que, embora a percepção sobre o risco individual de contrair uma doença hídrica seja elevada, ela não implica adoção de um comportamento preventivo. Uma possível explicação para esse resultado pode estar no conhecimento sobre os tipos de doenças veiculadas pela água e na percepção sobre os mecanismos de transmissão dessas doenças. A análise das respostas abertas auxiliará na identificação de possível viés otimista, de cunho heurístico, sobre a contaminação derivada da utilização.

\section{Percepções sobre a poluição do Rio Doce}

Aliada a fatores socioeconômicos, como distribuição de renda, condições gerais de saneamento ambiental, trabalho, moradia, escolaridade e outros, a qualidade do meio ambiente exerce uma influência direta no processo de saúde/doença das populações. Melhorias em um desses aspectos como, por exemplo, na qualidade dos recursos hídricos, podem gerar mudanças sig nificativas no perfil de saúde da comunidade, contribuindo para o seu desenvolvimento 31 . O contrário também pode ocorrer, ou seja, os efeitos da degradação dos recursos hídricos podem repercutir de maneira negativa sobre a saúde humana. Moraes \& Jordão 32 ressaltam que, no início dos anos 2000, a cada 14 segundos uma criança morria em decorrência de doenças hídricas e que $80 \%$ de todas as moléstias e mais de um terço dos óbitos dos países em desenvolvimento eram causados pelo consumo de água contaminada. Nesse sentido, a vigilância e o controle da qualidade da água para consumo

Estatísticas descritivas e testes bivariados de associação para as variáveis utilizadas na análise de regressão. Tumiritinga, Minas Gerais, Brasil, 2012.

\begin{tabular}{|c|c|c|c|c|c|}
\hline \multirow[t]{2}{*}{ Variáveis } & \multirow[t]{2}{*}{ Média } & \multirow[t]{2}{*}{ Desvio-padrão } & \multicolumn{2}{|c|}{ Amplitude } & \multirow{2}{*}{$\begin{array}{l}\text { Teste bivariado } \\
\text { (valor de } \mathrm{p} \text { ) }\end{array}$} \\
\hline & & & Mínimo & Máximo & \\
\hline Chance de contaminação & 2,25 & 1,12 & 0 & 4 & - \\
\hline Uso do Rio Doce para consumo & 0,81 & 0,39 & 0 & 1 & $0,04 *, * \star$ \\
\hline Familiar contraiu doença ao entrar no & 0,50 & 0,50 & 0 & 1 & $0,01 * \star \star \star *$ \\
\hline \multicolumn{6}{|l|}{ Rio Doce? } \\
\hline Sexo (masculino) & 0,30 & 0,46 & 0 & 1 & 0,55 * \\
\hline Idade & 45,11 & 16,82 & 18 & 98 & $0,10 * \star \star$ \\
\hline Renda domiciliar per capita & 537,11 & 548,93 & 0 & 4.000 & $0,18 * \star \star$ \\
\hline Reside há 1 ano ou menos & 0,11 & 0,31 & 0 & 1 & \multirow{4}{*}{0,25 * } \\
\hline Reside de 2-10 anos & 0,17 & 0,38 & 0 & 1 & \\
\hline Reside de 11-30 anos & 0,40 & 0,49 & 0 & 1 & \\
\hline Reside há mais de 30 anos & 0,32 & 0,47 & 0 & 1 & \\
\hline $\begin{array}{l}\text { Principal problema do rio: água suja e } \\
\text { esgoto }\end{array}$ & 0,29 & 0,46 & 0 & 1 & 0,19 * \\
\hline Distância da praia do Joá: 0-10 minutos & 0,52 & 0,50 & 0 & 1 & \multirow{4}{*}{$0,04 * * \star \star *$} \\
\hline Distância da praia do Joá: 11-20 minutos & 0,25 & 0,43 & 0 & 1 & \\
\hline Distância da praia do Joá: 21-30 minutos & 0,17 & 0,37 & 0 & 1 & \\
\hline Distância da praia do Joá: > 30 minutos & 0,06 & 0,24 & 0 & 1 & \\
\hline
\end{tabular}

* Teste qui-quadrado de Pearson;

** Valor de $\mathrm{p}<0,05$;

*** Teste $z$ (modelo probit ordenado simples).

Nota: associações e medidas descritivas calculadas sobre a amostra analítica final utilizada na regressão = 278 casos.

Fonte: dados primários (pesquisa amostral). 
Tabela 2

Estimativas da probabilidade de contaminação, uso e qualidade da água. Tumiritinga, Minas Gerais, Brasil, 2012.

\begin{tabular}{|c|c|c|c|c|c|c|}
\hline Variáveis & $\begin{array}{c}\text { Probit } \\
\text { ordenado I } \\
\text { (sem VI) }\end{array}$ & $\begin{array}{c}\text { Probit } \\
\text { ordenado I } \\
\text { (com VI) }\end{array}$ & $\begin{array}{c}\text { Probit } \\
\text { ordenado II } \\
\text { (sem VI) }\end{array}$ & $\begin{array}{c}\text { Probit } \\
\text { ordenado II } \\
\text { (com VI) }\end{array}$ & $\begin{array}{c}\text { Probit } \\
\text { Ordenado III } \\
\text { (sem VI) }\end{array}$ & $\begin{array}{c}\text { Probit } \\
\text { Ordenado III } \\
\text { (com VI) }\end{array}$ \\
\hline \multicolumn{7}{|l|}{ Variáveis estado } \\
\hline Uso da água do rio para consumo & 0,000 & $-1,618$ * & $-0,015$ & $-1,729 *$ & $-0,138$ & $-1,753$ * \\
\hline $0=$ não, $1=\operatorname{sim}$ & {$[0,184]$} & {$[0,313]$} & {$[0,196]$} & {$[0,289]$} & {$[0,214]$} & {$[0,467]$} \\
\hline Familiar infectado ao entrar no rio & 0,504 * & 0,527 * & 0,518 * & 0,495 * & 0,484 * & 0,477 * \\
\hline $0=$ não, $1=\operatorname{sim}$ & {$[0,114]$} & {$[0,107]$} & {$[0,116]$} & {$[0,127]$} & {$[0,123]$} & {$[0,130]$} \\
\hline \multicolumn{7}{|l|}{ Variáveis de controle } \\
\hline Sexo & & & $-0,104$ & $-0,201$ & $-0,189$ & $-0,188$ \\
\hline $0=$ feminino, $1=$ masculino & & & {$[0,131]$} & {$[0,139]$} & {$[0,144]$} & {$[0,142]$} \\
\hline Idade & & & $-0,003$ & $-0,003$ & $-0,004$ & $-0,007 * \star$ \\
\hline Contínua & & & {$[0,004]$} & {$[0,004]$} & {$[0,004]$} & {$[0,004]$} \\
\hline Renda per capita do domicílio & & & $-0,000$ & $-0,000$ & $-0,000$ & $-0,000$ \\
\hline Contínua & & & {$[0,000]$} & {$[0,000]$} & {$[0,000]$} & {$[0,000]$} \\
\hline \multicolumn{7}{|l|}{ Tempo contínuo de residência } \\
\hline \multicolumn{7}{|l|}{ Base $=1$ ano ou menos } \\
\hline Tempo contínuo de residência & & & $-0,202$ & $-0,221$ & $-0,182$ & $-0,282$ \\
\hline $2-10$ anos & & & {$[0,206]$} & {$[0,198]$} & {$[0,214]$} & {$[0,222]$} \\
\hline Tempo contínuo de residência & & & $-0,289 * \star$ & $-0,422 * \star \star$ & $-0,333 * \star$ & $-0,418 * \star \star$ \\
\hline $11-30$ anos & & & {$[0,171]$} & {$[0,168]$} & {$[0,180]$} & {$[0,196]$} \\
\hline Tempo contínuo de residência & & & $-0,274$ & $-0,407 * \star \star *$ & $-0,256$ & $-0,317$ \\
\hline Mais de 30 anos & & & {$[0,189]$} & {$[0,186]$} & {$[0,204]$} & {$[0,217]$} \\
\hline Principal problema ambiental do rio & & & & & 0,039 & $-0,019$ \\
\hline Poluição da água e esgoto = 1 & & & & & {$[0,147]$} & {$[0,144]$} \\
\hline \multirow[t]{2}{*}{ Tau 1} & $-1,597 *$ & $-2,835$ * & $-2,074$ * & $-3,594$ * & $-2,340$ * & $-3,765$ * \\
\hline & {$[0,213]$} & {$[0,274]$} & {$[0,304]$} & {$[0,345]$} & {$[0,350]$} & {$[0,435]$} \\
\hline \multirow[t]{2}{*}{ Tau 2} & $-0,196$ & $-1,702$ * & $-0,654$ * & $-2,436$ * & $-0,894$ * & $-2,604$ * \\
\hline & {$[0,186]$} & {$[0,299]$} & {$[0,279]$} & {$[0,346]$} & {$[0,317]$} & {$[0,508]$} \\
\hline \multirow[t]{2}{*}{ Tau 3} & 0,281 & $-1,235$ * & $-0,175$ & $-1,958$ * & $-0,386$ & $-2,126$ * \\
\hline & {$[0,185]$} & {$[0,312]$} & {$[0,279]$} & {$[0,353]$} & {$[0,315]$} & {$[0,531]$} \\
\hline \multirow[t]{2}{*}{ Tau 4} & 1,412 * & $-0,180$ & 0,962 * & $-0,918 * * \star$ & 0,731 * & $-1,088 * *$ \\
\hline & {$[0,192]$} & {$[0,340]$} & {$[0,283]$} & {$[0,375]$} & {$[0,317]$} & {$[0,577]$} \\
\hline \multirow[t]{2}{*}{ Atanhrho 12} & & 1,289 * & & $1,398 * \star * *$ & & 1,484 ** \\
\hline & & {$[0,378]$} & & {$[0,381]$} & & {$[0,784]$} \\
\hline Teste de Endogenia de Hausman & 43,12 & 0,0000 & 39,84 & 0,0000 & 33,18 & 0,0000 \\
\hline Observações & & 278 & & 278 & & 278 \\
\hline
\end{tabular}

${ }^{*} \mathrm{p}<0.01$

$* * p<0,1$

$* * * p<0,05$.

Nota: erros-padrão robustos entre colchetes.

Fonte: dados primários (pesquisa amostral).

são fundamentais para a prevenção das doenças relacionadas com a água 22 .

Os resultados revelam que a maior parte dos entrevistados tem consciência da poluição do
Rio Doce e de algumas fontes potenciais de tal poluição. Os problemas mais graves, para uma grande parcela, resultam das ações de indústrias localizadas na região, as quais contaminam 
a água ao lançarem produtos químicos no rio. Alguns entrevistados também ressaltaram o problema da poluição do rio como decorrente do lançamento de resíduos domésticos.

"Pelo seguinte, temos indústrias jogando produto químico, e tem as represas que juntam coisas no fundo. Há pouco tempo a água tava com gosto horrível" (Entrevistado 10). "Por causa da sujeira mesmo, esgoto. Tudo trem que é ruim eles jogam ali" (Entrevistado 34).

Adicionalmente, a percepção de alguns entrevistados é de que a poluição do rio também está relacionada ao fato dele ser um local de desova de corpos.

"Porque tem tanta coisa que desce. Mês de novembro acharam uma mulher no saco plástico dentro do rio" (Entrevistado 12).

Saber que o Rio Doce é poluído e quais os agentes causadores da poluição não significa, contudo, conhecer as doenças que podem ser veiculadas por águas contaminadas e, muito menos, entender a gravidade de muitas dessas doenças. Para McClain et al. 33, compreender como as pessoas envolvidas percebem o risco associado com as doenças provenientes da água, e suas motivações para adotar comportamentos preventivos, é fundamental para que se possa prevenir a difusão de doenças hídricas.

\section{As doenças advindas do Rio Doce}

Informações extraídas do Sistema de Informação de Agravos de Notificação (SINAN; http:// dtr2004.saude.gov.br/sinanweb/index.php, acessado em 03/Set/2014), do Departamento de Informática do SUS (DATASUS), sugerem que em Tumiritinga os números de registros de casos confirmados notificados, entre 2010 e 2014, para as doenças de veiculação hídrica, tais como hepatites virais e esquistossomose, foram baixos (3 e 13 casos, respectivamente). Para leptospirose, difteria, cólera ou febre tifoide não houve casos confirmados no mesmo período. Há, provavelmente, subenumeração de casos, uma vez que os dados amostrais apontam para a ocorrência de doenças de veiculação hídrica em residentes de Tumiritinga adquiridas em função de terem entrado no Rio Doce. Por exemplo, dos 173 casos reportados de alguma doença de veiculação hídrica (entre os 352 entrevistados), 126 reportaram casos de doenças de pele $(72,8 \%), 11$ contração de esquistossomose $(6,4 \%), 1$ febre tifoide $(0,6 \%)$ e 32 relataram casos denominados como "outras doenças não especificadas” (18,5\%). Adicionalmente, 3 pessoas relataram não saber o nome da doença contraída (1,7\%). O reconhecimento dessas doenças oriundas do uso do Rio Doce reflete, inclusive, na alta classificação percebida de risco de contaminação pelo uso do rio (média de 2,24 numa escala de 0 - sem possibilidade a 4 - enorme).

A despeito do reconhecimento da alta chance de contaminação ao usar as águas poluídas do Rio Doce, poucos sabem sobre como e por quais doenças podem ser contaminados. Uma preocupação mais acentuada aparece em relação à leptospirose.

"Porque são de deixar mancha na gente, já é também uma contaminação da xistosa" (Entrevistado 47). "Já vi muita gente pegar mancha e dar dor nos ossos" (Entrevistado 10). "Porque eles falam muito das doenças de rato, e as enchentes traz tudo que era nojerada, esgoto" (Entrevistado 64).

Os resultados também revelam desinformação acerca da transmissão de outras doenças, que não são de veiculação hídrica. Para alguns entrevistados, a AIDS, por exemplo, pode ser contraída porque muitas pessoas jogam preservativos utilizados no rio.

"Por lixo de outras cidades e daqui mesmo. $O$ povo que frequenta joga lixo, tem o risco até de pegar Aids pelo tanto de camisinha" (Entrevistado 220).

Apesar de perceberem o rio como um local poluído e fonte de transmissão de doenças, muitos entrevistados alegaram que continuam entrando no rio. Uma das possíveis explicações para esse comportamento pode estar na percepção de risco que possuem.

\section{Entrar ou não no Rio Doce?}

McClain et al. 33 definem risco como a probabilidade de uma perda ou de um resultado adverso, constituído por dois elementos: a probabilidade que um resultado adverso ocorra e a severidade deste resultado. Eles alegam, no entanto, que pessoas leigas geralmente têm uma definição de risco intuitiva, normalmente baseada em suas percepções de probabilidade de resultados negativos, de controle da situação e de informação disponível sobre o risco. Alguns extratos de falas sugerem que muitos entrevistados ainda possuem informações equivocadas ou insuficientes acerca da possibilidade de contraírem algum tipo de doença quando utilizam o rio, o que reduz a motivação para a adoção de comportamento preventivo em relação ao uso do rio.

“Na água não pega nada não, porque o Rio tem muitos peixes que comem a sujeira" (Entrevistado 343). "Água corrente não corre risco" (Entrevistado 88).

Nordgren et al. 34 também destacam o papel da familiaridade que os indivíduos têm com o risco ao qual estão expostos e da expectativa de 
tempo que possuem em relação ao resultado, ou seja, se o resultado da exposição será imediato ou acontecerá em longo prazo. Uma das falas apresentadas ilustra a questão da familiaridade que os entrevistados têm com um dos resultados adversos da exposição às águas contaminadas do Rio Doce. Bastante conhecida na região, a esquistossomose parece não ser percebida como uma doença de sérias consequências. Nesse sentido, é possível argumentar que o entendimento da extensão do risco resulta em uma separação entre as doenças transmitidas pelo rio e o uso do mesmo. Adicionalmente, como sugerido pela teoria da controlabilidade 6,34, a sensação de controle sobre determinada situação, em geral, resulta na diminuição da percepção de risco, o que leva muitos indivíduos a se exporem à situação que gerará um resultado adverso.

"Eu já dei muita xistose, já fui proibida, mas sou teimosa e tomo banho assim mesmo" (Entrevistada 345).

Os resultados sugerem que a relação entre percepção de risco e comportamento preventivo é muito tênue. Questões atinentes à disponibilidade de informação, à cultura local e a comportamentos já consolidados em relação ao uso do rio podem influenciar a intensidade da ligação entre percepção de risco e comportamento preventivo na região. $\mathrm{O}$ aparente paradoxo encontrado parece ser predominantemente explicado pelo viés otimista heurístico sobre a percepção da chance de contaminação, como sugerido por Bateman et al. 14, sobrepondo a influência da controlabilidade, como sugerido por Weinstein 6 .

\section{Considerações finais}

Os resultados deste trabalho apontam para um aparente paradoxo na relação entre o uso do Rio Doce e a percepção sobre chances de contaminação decorrente do seu uso. A teoria psicológica sobre heurística do otimismo argumenta que esse paradoxo advém da falha de interpretação dos mecanismos geradores do risco, reduzindo a chance dos indivíduos engajarem-se em comportamento preventivo. De fato, os resultados da regressão concordam com essa interpretação, pois pessoas que usam o rio para consumo são as que classificam o risco de contaminação como mais baixo, mesmo após ser atenuado o efeito da endogeneidade por simultaneidade entre percepção de risco e utilização. Embora esse resultado em si não seja capaz de informar sobre as causas, os resultados qualitativos sugerem que os moradores de Tumiritinga não compreendem plenamente os mecanismos de transmissão das doenças veiculadas pela água. Assim, vários discursos reconhecem a existência da poluição e da contaminação, mas sugerem imunidade.

Os moradores apresentam várias razões, como a água corrente e a existência de peixes, como fatores de protetores. A presença da Prainha do Jaó, a praia de rio do município resultante do longo processo de assoreamento do rio, pode ser um dos fatores que afetam a percepção sobre o risco de contaminação pelo contato com as águas do Rio Doce, uma vez que a representação social formada em relação à prainha evoca sentimentos eminentemente positivos. Por fim, verificou-se um efeito significativo de ter alguém na família contaminado pelas águas do rio sobre a chance de contaminação, sinalizando um componente afetivo na percepção do risco, conforme sugerem Bateman et al. 14. Curiosamente, embora os resultados da regressão apontem para um efeito estatisticamente significativo em relação a ter algum parente contaminado sobre a percepção de risco de contaminação, alguns discursos sugerem que a percepção não é suficiente para impedir as pessoas de continuarem utilizando o Rio, reforçando a ideia de que o desconhecimento sobre os reais mecanismos de transmissão de doenças hídricas é a causa principal que explica a associação positiva entre alto risco percebido e uso frequente. Ademais, frequentar a Praia do Jaó, que expõe os indivíduos ao risco, é visto como uma entidade separada, evocando imagens positivas. Essa separação entre a praia de rio e o rio na representação social dos residentes de Tumiritinga cria um desafio adicional para a promoção de comportamento preventivo quanto às práticas de utilização dos recursos hídricos na região.

Os resultados sugerem atenção para a alteração futura do risco epidemiológico em Tumiritinga, uma vez que existe um componente voluntário não consciente, para além dos riscos gerados pelas enchentes. A subestimação do risco só parece ser atenuada entre os que têm pessoas próximas diretamente afetadas. Nesse sentido, estratégias adotadas em práticas de educação ambiental parecem ser de fundamental importância para que os moradores de Tumiritinga e usuários do Rio Doce entendam não somente a extensão do risco a que estão expostos quando entram em suas águas, mas que possam, a partir das experiências diretas e indiretas, adotar comportamentos menos adversos em termos de saúde.

Por fim, cabe pontuar que os resultados encontrados para Tumiritinga podem exprimir a situação de muitas outras pequenas comunidades brasileiras que vivem próximas de cursos de água e os utilizam tanto para o consumo quanto 
para o lazer. Novos dados amostrais, relativos ao mesmo projeto de pesquisa do qual este artigo é derivado, estão em fase de coleta e processamento para os municípios de Galiléia e Governador Valadares. A comparação dos resultados aqui obtidos com as novas amostras em outros municípios da região poderá contribuir para a generalização das evidências de falha heurística na associação entre uso e percepção de risco relativo a recursos hídricos contaminados.

\section{Resumen}

La fuerte relación que los residentes tienen con Río Doce, así como con sus inundaciones regulares, representan factores de exposición continua para el riesgo de enfermedades transmitidas por el agua. Este estudio examina la asociación entre la percepción de la contaminación y el uso del río, así como los mecanismos heurísticos usados en la formación de la percepción del riesgo. Se utilizó un modelo probit ordenado con variable instrumental y análisis de redes temáticas aplicadas a una base de datos primaria, representativa de los residentes de Tumiritinga, Minas Gerais, Brasil, para el año 2012. Los resultados indican que, aunque la mayoría de los residentes (92,6\%) se dan cuenta del riesgo de contraer algún tipo de enfermedad, cuando se nada en Río Doce, sólo el 11,4\% declara no entrar en el agua. El análisis de contenido sugiere que esta paradoja se debe a la falta de comprensión de la población de los mecanismos de transmisión de enfermedades hídricas, creándose un sesgo optimista sobre las posibilidades de contaminación.

Riesgos Ambientales; Contaminación del Agua; Enfermedades; Transmitidas por el Agua

\section{Colaboradores}

G. R. Guedes participou da concepção do estudo, revisão da literatura, coleta de dados, análise e interpretação dos resultados quantitativos e redação do manuscrito. A. B. Simão participou da concepção do estudo, revisão da literatura, análise e interpretação dos resultados qualitativos e redação do manuscrito. C. A. Dias e E. O. Braga contribuíram com a operacionalização da pesquisa, participou da coleta de dados e da redação do manuscrito.

\section{Agradecimentos}

Este estudo contou com o apoio financeiro da FAPEMIG (Edital 01/2011 - Demanda Universal - Processo no SHA - APQ-01807-11, Edital 01/2012 - Demanda Universal - Processo no CSA - APQ-00244-12) e do CNPq (Edital Universal 14/2012 - Processo no 483714/2012-7). Agradecemos também aos parecerias anônimos de $\mathrm{Ca}$ dernos de Saúde Pública pelos comentários e sugestões feitas em versões anteriores a esta. 


\section{Referências}

1. Prüss A, Kay D, Fewtrell L, Bartram J. Estimating the burden of disease from water, sanitation, and hygiene at a global level. Environ Health Perspect 2002; 110:537-42.

2. Hamner S, Tripathi A, Mishra RK, Bouskill N, Broadway SC, Pyle BH, et al. The role of water use patterns and sewage pollution in incidence of water-borne/enteric diseases along the Ganges river in Varanasi, India. Int J Environ Health Res 2006; 16:113-32.

3. Singley JA. Hazard versus risk. Journal of Chemical Health and Safety 2004; 11:14-6.

4. van Westen CJ, van Asch TW, Soeters R. Landslide hazard and risk zonation - why is it still so difficult? Bulletin of Engineering Geology and the Environment 2006; 65:167-84.

5. Soares SRA, Bernardes RS, Cordeiro Netto OM Relações entre saneamento, saúde pública e meio ambiente: elementos para formulação de um modelo de planejamento em saneamento. Cad Saúde Pública 2002; 18:1713-24.

6. Weinstein ND. Why it won't happen to me: perceptions of risk factors and susceptibility. Health Psychol 1984; 3:431-57.

7. Chambers JR, Windschitl PD. Biases in social comparative judgments: the role of nonmotivated factors in above-average and comparative-optimism effects. Psychol Bull 2004; 130:813-38.

8. Wit JBF, Das E, Vet R. What works best: objective statistics or a personal testimonial? An assessment of the persuasive effects of different types of message evidence on risk perception. Health Psychol 2008; $27: 110-5$.

9. Slovic P. The more who die, the less we care. In: Michel-Kerjan E, Slovic P, editors. The irrational economist: making decisions in a dangerous world. New York: Public Affair; 2010. p. 30-40.

10. Deery HA. Hazard and risk perception among young novice drivers. J Safety Res 2000; 30:225-36.

11. Schwarzer R. Social-cognitive factors in changing health-related behaviors. Curr Dir Psychol Sci 2001; 10:47-51.

12. Baruch F, Slovic P, Lichtenstein S, Read S, Combs B. How safe is safe enough? A psychometric study of attitudes toward technological risks and benefits. In: Slovic P, editor. The perception of risk. New York: Routledge; 2000. p. 80-103.

13. Peters EM, Burraston B, Mertz CK. An emotionbased model of risk perception and stigma susceptibility: cognitive appraisals of emotion, affective reactivity, worldviews, and risk perceptions in the generation of technological stigma. Risk Anal 2004; 24:1349-67.
14. Bateman I, Dent S, Peters E, Slovic P, Starmer C. The affect heuristic and the attractiveness of simple gambles. In: Slovic P, editor. The feeling of risk: new perspectives on risk perception. New York: Routledge; 2010. p. 3-19.

15. Slovic P. If I look at the mass, I shall never act psychic numbing and genocide. Judgment and Decision Making 2007; 2:79-95.

16. Morua AR, Halvorsen KE, Mayer AS. Waterborne disease-related risk perceptions in the Sonora River Basin, Mexico. Risk Anal 2011; 31:866-78.

17. Eaton L, Flisher AJ, Aarø LE. Unsafe sexual behaviour in South African youth. Soc Sci Med 2003; 56:149-65.

18. Li X, Stanton B, Fang X, Lin D, Mao R, Wang J, et al HIV/STD risk behaviors and perceptions among rural-to-urban migrants in China. AIDS Educ Prev 2004; 16:538-56.

19. Corral-Verdugo V, Frías-Armenta M, GonzálezLomelí D. Percepción de riegos, conducta proambiental y variables demográficas en una comunidad de Sonora, México. Región y Sociedad 2003; 15:49-72.

20. Luiz OC, Cohn A. Sociedade de risco e risco epidemiológico. Cad Saúde Pública 2006; 22:2339-48.

21. Cavalcanti BS. Gestão integrada de recursos hídricos e do meio ambiente: medidas institucionais no Brasil no contexto da reforma do Estado. Revista de Administração Pública 2013; 28:162-73.

22. Secretaria de Vigilância em Saúde, Ministério da Saúde. Vigilância e controle da qualidade da água para consumo humano. Brasília: Ministério da Saúde; 2006. (Série B. Textos Básicos de Saúde).

23. d'Aguila PS, Roque OCC, Miranda CAS, Ferreira AP. Avaliação da qualidade de água para abastecimento público do Município de Nova Iguaçu. Cad Saúde Pública 2000; 16:791-8.

24. Comitê da Bacia Hidrográfica do Rio Doce. PHIR DOCE - Plano integrado de recursos hídricos da bacia hidrográfica do Rio Doce e planos de ações para as unidades de planejamento e gestão de recursos hídricos no âmbito da bacia do Rio Doce. v. I; 2010. http://www.riodoce.cbh.gov.br/ docs/ planobacia/PIRH/PIRH_Doce_Volume_I.pdf (acessado em 24/Fev/2014).

25. Espindola HS. Sertão do Rio Doce. Governador Valadares: Editora Univale; 2005.

26. Hora AM. Representações sociais da comunidade de Tumiritinga sobre o Rio Doce e a Prainha do Jaó [Dissertação de Mestrado]. Governador Valadares: Universidade Vale do Rio Doce; 2013.

27. Coelho MAT. Rio Doce: a espantosa evolução de um vale. Belo Horizonte: Autêntica Editora; 2011. 
28. Groves RM, Fowler FJ, Couper MP, Lepkowski JM, Singer E, Tourangeau R. Survey methodology. 2nd Ed. Hoboken: Wiley-Interscience; 2009.

29. Wooldridge JM. Econometric analysis of cross section and panel data. Cambridge/London: The MIT Press; 2010.

30. Attride-Stirling J. Thematic networks: an analytic tool for qualitative research. Qualitative Research 2001; 1:385-405.

31. Rodrigues ASL, Malafaia G. Degradação dos recursos hídricos e saúde humana: uma atualização. Revista Saúde e Ambiente 2009; 10:13-23.
32. Moraes DSL, Jordão BQ. Degradação de recursos hídricos e seus efeitos sobre a saúde humana. Rev Saúde Pública 2002; 36:370-4.

33. McClain J, Bernhardt JM, Beach MJ. Assessing parents' perception of children's risk for recreational water illnesses. Emerg Infect Dis 2005; 11:670-6.

34. Nordgren LF, van der Pligt J, van Harreveld F. Unpacking perceived control in risk perception: the mediating role of anticipated regret. J Behav Decis Mak 2007; 20:533-44.

Recebido em 22/Abr/2014

Versão final reapresentada em 08/Set/2014 Aprovado em 09/Jan/2015 